\title{
Ultrasonic Preparation of Emulsions Derived from Aqueous Bio-oil Fraction and 0\# Diesel and Combustion Characteristics in Diesel Generator
}

\author{
Yuping Li, Tiejun Wang, * Wei Liang, Chuangzhi Wu, Longlong Ma, Qi Zhang, Xinghua Zhang, and Ting Jiang \\ Key Laboratory of Renewable Energy and Gas Hydrate, Guangzhou Institute of Energy Conversion, Chinese Academy of Sciences, \\ Guangzhou 510640, People's Republic of China
}

Received October 2, 2009. Revised Manuscript Received January 18, 2010

\begin{abstract}
Preparation of emulsions from aqueous fractions of biomass pyrolysis liquid (bio-oil) and 0\# diesel was studied under ultrasonic power. The thermogravimetric analysis and combustion characteristics of the optimized emulsions in a particular diesel generator were also investigated. The results show that the mass ratio of aqueous bio-oil fraction to $0 \#$ diesel affected the emulsion stability greatly, by factor analysis method, and the optimized emulsion was not stratified for 30 days when the ratio was 1:9 under the following conditions: water:bio-oil mass ratio, 2:1; ultrasonic time, $120 \mathrm{~min}$; formula emulsifier addition (Span-80:Tween-80:Tween-20: $n$-octanol ratio $=72: 9: 9: 10$, wt \%), 3 wt \% (which was longer than that prepared by mechanical agitation). The density and heat value of the nonstratified emulsions (after 30 days) were similar with those of $0 \#$ diesel, because of the low aqueous bio-oil fraction content and $0 \#$ diesel dilution; however, an increase of more than $38.4 \%$ in the dynamic viscosity and a decrease in $\mathrm{pH}$ from 5.26 to $<3$ may result from the concentrated polar oxygenates and carbohydrates in aqueous bio-oil fraction, which were further solubilized in water-in-oil (W/O) emulsion droplets. In addition, ultrasonic emulsification resulted in entropy augmentation and equilibrium tendencies (by thermodynamic analysis). Thermogravimetric analysis of the emulsion shows that there is a weight loss peak that is attributed to residual carbon combustion above $400{ }^{\circ} \mathrm{C}$, in addition to a similar hydrocarbon vaporization peak at $180{ }^{\circ} \mathrm{C}$ with diesel. The existence of residual carbon and the high viscosity of the emulsion might have caused inefficient combustion when the output power of diesel generator was above $1400 \mathrm{~W}$ with high feeding amount, which also resulted in higher exhaust gas temperature than diesel feedstock, although microexplosion may have occurred. Moreover, when the output power increased to $1600 \mathrm{~W}$ with the emulsion used, the $\mathrm{O}_{2}$ and $\mathrm{NO}_{\mathrm{X}}$ emissions decreased $21 \%$ and $87.2 \%$, respectively, the $\mathrm{CO}_{2}$ emission increased $13.3 \%$, and the $\mathrm{CO}$ and $\mathrm{SO}_{2}$ emissions increased quickly to $5124 \mathrm{ppm}$ and $59 \mathrm{ppm}$, respectively, compared with pure diesel. The corrosion and blockage caused by coking of the injector were obvious.
\end{abstract}

\section{Introduction}

Fast pyrolysis of biomass residues to bio-oil is an competitive biomass utilization technology to substitute fossil fuels with a high bio-oil yield rate of $70 \%-80 \%{ }^{1,2}$ Several pilot bio-oil production demonstrations have been developed or commercialized, such as Ensyn Technologies (six circulating fluidized-bed plants, the largest of which has a capacity of $50 \mathrm{t} /$ day), Dynamotive (operating a $10 \mathrm{t} /$ day fluidized-bed process; currently building a $100 \mathrm{t}$ /day plant), BTG (operating a rotary cone reactor with a capacity of $5 \mathrm{t} /$ day; wants to build a $50 \mathrm{t}$ /day plant). ${ }^{3}$ However, the direct use of bio-oil poses significant challenges, because of low $\mathrm{pH}$, low cetane value, high ash and water content, high viscosity, and high operating temperatures, limiting wide application in decentralized

*To whom correspondence should be addressed. Phone: +86-2087057751. Fax: +86-20-87057731. E-mail: wangtj@ms.giec.ac.cn.

(1) Demirbas, A. Biomass resource facilities and biomass conversion processing for fuels and chemicals. Energy Convers. Manage. 2001, 42 (11), 1357-1378.

(2) Czernik, S.; Bridgwater, A. V. Overview of Applications of Biomass Fast Pyrolysis Oil. Energv Fuels 2004, 18 (2), 590-598.

(3) Huber, G. W.; Iborra, S.; Corma, A. Synthesis of transportation fuels from biomass: Chemistry, catalysts, and engineering. Chem. Rev. 2006, 106 (9), 4044-4098

(4) Zhang, Q.; Chang, J.; Wang, T. J.; Xu, Y. Review of biomass pyrolysis oil properties and upgrading research. Energy Convers. Manage. 2007, 48 (1), 87-92. co-generation and power production systems. ${ }^{4,5}$ In particular, the engine injection system must be modified (e.g., additional plug and spark ignition) to suit the deleterious properties of pure bio-oil feedstock. ${ }^{6}$ Emulsification of bio-oil with diesel represents an interesting possibility that allows the direct usage of bio-oil in the engine without additional modification costs with regard to engineering and manufacturing., Many previous studies were devoted to infer the major features exhibited by bio-oil during emulsification with diesel and long-term behavior in engines. ${ }^{9}$ Yet, the existing

(5) Chiaramonti, D.; Oasmaa, A.; Solantausta, Y. Power generation using fast pyrolysis liquids from biomass. Renew. Sustainable Energy Rev. 2007, 11 (6), 1056-1086.

(6) Karthikeyan, R.; Mahalakshmi, N. V. Performance and emission characteristics of a turpentine-diesel dual fuel engine. Energy 2007, 32 (7), 1202-1209.

(7) Chiaramonti, D.; Bonini, M.; Fratini, E.; Tondi, G.; Gartner, K.; Bridgwater, A. V.; Grimm, H. P.; Soldaini, I.; Webster, A.; Baglioni, P. Development of emulsions from biomass pyrolysis liquid and diesel and their use in engines-Part 1: emulsion production. Biomass Bioenergy 2003, 25 (1), 85-99.

(8) Garcia-Perez, M.; Adams, T. T.; Goodrum, J. W.; Geller, D. P.; Das, K. C. Production and Fuel Properties of Pine Chip Bio-oil/ Biodiesel Blends. Energv Fuels 2007, 21 (4), 2363-2372.

(9) Baglioni, P.; Chiaramonti, D.; Bonini, M.; Soldaini, I.; Tondi, G. Bio-Crude-Oil/Diesel Oil Emulsification: Main Achievements of the Emulsification Process and Preliminary Results of Tests on Diesel Engine. In Progress in Thermochemical Biomass Conversion; Bridgwater, A. V., Ed.; Blackwell Science, Ltd.: Oxford, U.K., 2008; pp 1525-1539. 
limitations, such as product quality (emulsion destabilization, e.g., stratification, breaking), and technological adaptations (such as corrosion of the injector nozzle) still impede the full development of the biomass pyrolysis-to-energy chain. $^{10}$

Recently, multiseparation of bio-oil by adding water to an aqueous fraction (mostly oxygenate compounds, such as alcohols, phenols, aldehydes, ketones, acids and esters, and carbohydrates in water) and an organic compound fraction (lignin-derived oligomers) to different applications has become an new research focus, ${ }^{11-14}$ especially on converting the aqueous, carbohydrate (or oxygenate)-derived fraction to hydrogen by steam reforming. Ikura also obtained light fractions of bio-oil by centrifugation and emulsified them in 2\# diesel by CANMET emulsifier, which decreased the viscosity and corrosiveness. ${ }^{15}$ Emulsification of the lesscomplex water-soluble fraction with diesel and combustion of the emulsions in boilers, turbines, and engine are supposed to enhance the fuel stability and decrease the corrosion and blockage of the engine subassemblies, caused by polycondensation and thermal degradation of pure bio-oil/diesel emulsion. ${ }^{8,11}$ Yet, few concrete experiments have been reported in this field.

The objective of this paper is to report the experimental study of the emulsification of the aqueous bio-oil fraction with 0\# diesel under ultrasonic conditions and the combustion characteristics of the emulsion in diesel generator. The effects of influence factors such as the bio-oil:water mass ratio, the aqueous bio-oil fraction:diesel mass ratio, the emulsifier time, and ultrasound time on emulsion stability, as well as the emulsification mechanism, were primarily studied and concluded. Moreover, the combustion characteristics, operation stability, and exhaust emission of the optimized emulsion in a Model CP2500CL diesel generator (Chongqing Camel Power Machinery Co., Ltd., Chongqing, PRC) were also discussed to obtain useful information regarding bio-oil applications.

\section{Experimental Section}

2.1. Preparation of Aqueous Bio-oil Fraction, Emulsifier, and Aqueous Bio-oil Fraction/Diesel Emulsion. Bio-oil is produced from the fast pyrolysis of pine sawdust (60-80 mesh, $2.5 \mathrm{~kg} / \mathrm{h})$ at $450-550{ }^{\circ} \mathrm{C}$ in a fluidized-bed reactor $131 \mathrm{~cm}$ in height and $12 \mathrm{~cm}$ in diameter with preheated $\mathrm{N}_{2}\left(300{ }^{\circ} \mathrm{C}, 3 \mathrm{~m}^{3} / \mathrm{h}\right)$ as fluidizing gas, pumped from the reactor bottom. The cleaned hot gas, after cyclones, was condensed using an ice-water bath in

(10) Chiaramonti, D.; Bonini, M.; Fratini, E.; Tondi, G.; Gartner, K.; Bridgwater, A. V.; Grimm, H. P.; Soldaini, I.; Webster, A.; Baglioni, P. Development of emulsions from biomass pyrolysis liquid and diesel and their use in engines-Part 2: Tests in diesel engines. Biomass Bioenergv 2003, $25(1), 101-111$.

(11) Scholze, B.; Meier, D. Characterization of the water-insoluble fraction from pyrolysis oil (pyrolytic lignin). Part I. PY-GC/MS, FTIR, and functional groups. J. Anal. Appl. Pvrolvsis 2001, 60 (1), 41-54.

(12) Kechagiopoulos, P. N.; Voutetakis, S. S.; Lemonidou, A. A.; Vasalos, I. A. Hydrogen Production via Steam Reforming of the Aqueous Phase of Bio-Oil in a Fixed Bed Reactor. Energv Fuels 2006, 20 (5), 2155-2163.

(13) Vagia, E. C.; Lemonidou, A. A. Thermodynamic analysis of hydrogen production via autothermal steam reforming of selected components of aqueous bio-oil fraction. Int. J. Hydrogen Energy 2008, 33 (10), 2489-2500.

(14) Basagiannis, A. C.; Verykios, X. E. Steam reforming of the aqueous fraction of bio-oil over structured $\mathrm{Ru} / \mathrm{MgO} / \mathrm{Al}_{2} \mathrm{O}_{3}$ catalysts. Catal. Todav 2007, 127 (1-4), 256-264.

(15) Ikura, M.; Stanciulescu, M.; Hogan, E. Emulsification of pyrolysis derived bio-oil in diesel fuel. Biomass Bioenergy 2003, 24 (3), 221232. the condenser to collect liquid product, as described in ref 16 . The elemental composition of the bio-oil was given as follows: C, 53.6; H, 5.95; O, 40.3; N, 0.02; S, 0.13 (water free, wt \%) with a water content of $35.5 \mathrm{wt} \%$. The abundant chemical compositions were 1-hydroxy-2-propanone $(10.1 \%)$ and acetic acid $(5.62 \%)$. It was separated into an aqueous phase (predominantly carbohydrate-derived) and an organic phase (ligninderived) by adding water to the bio-oil at weight ratios of 2:1, $1: 1$, and $1: 2$. After mixing, incubating for $24 \mathrm{~h}$, the upper aqueous fractions were used to make emulsions and denoted as $\mathrm{Ba} 1, \mathrm{Ba} 2$, and $\mathrm{Ba} 3$, respectively. The $0 \#$ diesel was available commercially and is abbreviated as diesel below.

Two formula emulsifiers were prepared by mixing nonionic surfactants of Span-80, Tween-80, Tween-20, TritonX-100, and n-octanol at mass ratios of 72:9:9:0:10 and 63:9:9:9:10 (denoted as Mix1 and Mix2, respectively).

The water-in-oil $(\mathrm{W} / \mathrm{O})$ emulsions are prepared by adding the formula emulsifier to diesel and then adding the aqueous bio-oil fraction to the resulting mixture using an ultrasonic transducer (15 kHz center frequency, $10 \mathrm{~mm}$ in diameter, Model SN-D-201000 Sinuo Inc., Guangzhou, PRC) immersed into the liquid for a long time (120 min) to obtain a clear single phase. In addition, the aqueous bio-oil fraction/diesel emulsion was denoted as $\mathrm{ABF} / \mathrm{D}$. The freshly prepared emulsions were poured into burets and incubated at room temperature to measure the amount of phase separation of the water phase from the oil phase. The stratification rates were defined by the volume ratios of the dense lower-phase volume, relative to that of the entire liquid.

2.2. Physicochemical Characterization of ABF/D Emulsions. The dynamic viscosity was measured with Model WFY-108D viscometers (GB/T265-1988). The gross calorific value (high heating value, HHV) was measured using a Model WGR-1 calorimetric bomb (ASTM Standard D4809). The lower heating value (LHV) was calculated with elemental analysis data by subtracting the contribution of the water content from the HHV. The acidity was evaluated using a Model PHS-3C precision $\mathrm{pH}$-meter (Shanghai REX Instrument Factory). The density was measured using a capillary stoppered pycnometer (Model GB/T1337-1992). The water content was determined via Karl Fischer titration (ASTM Standard D1744, Model GB11146-89), using a Metrohm Model 787 KF Titrino system, and the elemental contents of carbon, hydrogen, oxygen, nitrogen, and sulfur were measured with a Vario EL Analyzer. ${ }^{17}$ The thermogravimetric (differential thermogravimetry, DTG) analysis results were obtained using a Model STA449FC Jupitor-FTIR Tensor 27 system from $40{ }^{\circ} \mathrm{C}$ to $1100{ }^{\circ} \mathrm{C}$ at the heating rate of $20{ }^{\circ} \mathrm{C} / \mathrm{min}$ in air with an online FTIR spectrometer to determine the tailgas component.

2.3. $\mathrm{ABF} / \mathrm{D}$ Emulsion Combustion in Diesel Generator and Operation Procedure. The optimized ABF/D emulsion was introduced to the Model CP2500CL diesel generator $(2000 \mathrm{~W}$ of full output power, made by Chongqing Camel Power Machinery Co., Ltd., Chongqing, PRC; single-cylinder, naturally aspirated, four-stroke, air-cooled, direct-injection diesel engine with a piston displacement of $211 \mathrm{~mL}$ ) with an electric current meter and 10 incandescent lamps (200 W each) to determine the generator output power $\left(P_{\mathrm{e}}\right)$ in Figure 1. The generator was operated at a constant injection timing of $17^{\circ} \mathrm{CA}$ BTDC at different $P_{\mathrm{e}}$ values from $200 \mathrm{~W}$ to $1800 \mathrm{~W}$ with an increment of $200 \mathrm{~W}$ at $3000 \mathrm{rpm}$. In each run, the diesel generator operated with no load for $5 \mathrm{~min}$, and then switched to different $P_{\mathrm{e}}$ values for more than $30 \mathrm{~min}$. The exhaust gas from the generator was determined using a portable infrared gas analyzer (Model TESTO350) every $2 \mathrm{~min}$, and the

(16) Zheng, J. L.; Zhu, X. F.; Guo, Q. X.; Zhu, Q. S. Thermal conversion of rice husks and sawdust to liquid fuel. Waste Manage. 2006, 26 (12), 1430-1435.

(17) Zhang, Q.; Chang, J.; Wang, T. J.; Xu, Y. Upgrading Bio-oil over Different Solid Catalysts. Energv Fuels 2006, 20 (6), 2717-2720. 


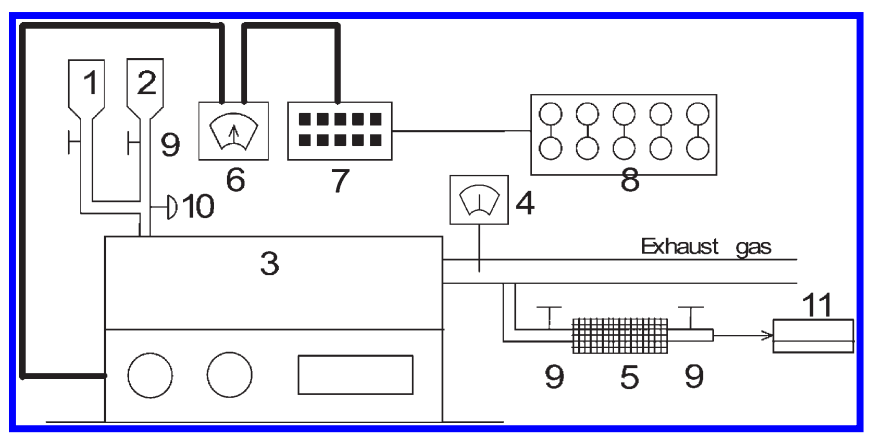

Figure 1. Schematic diagram of $\mathrm{ABF} / \mathrm{D}$ emulsion combustion in a Model CP2500CL diesel generator. (Legend: 1, diesel tank; 2, emulsion tank; 3, diesel generator; 4, temperature detector; 5, filter; 6, electric current meter; 7, switch controller; 8, incandescent lamps; 9, ball valve; 10, T-type valve; and 11, portable infrared gas analyzer).

Table 1. Physical Properties of the Aqueous Bio-oil Fraction

\begin{tabular}{lllll}
\hline \multicolumn{1}{c}{ physical property } & \multicolumn{1}{c}{ Ba1 } & \multicolumn{1}{c}{ Ba2 } & \multicolumn{1}{c}{ Ba3 } & \multicolumn{1}{c}{ bio-oil } \\
\hline mass ratio of water to bio-oil & $2: 1$ & $1: 1$ & $1: 2$ & 0 \\
water content $(\mathrm{wt} \%)$ & 80.1 & 72.6 & 61.4 & 35.5 \\
density $(\mathrm{g} / \mathrm{mL})$ & 1.094 & 1.118 & 1.152 & 1.224 \\
lower heating value, & N/A & N/A & N/A & 13.2 \\
$\quad$ LHV $(\mathrm{MJ} / \mathrm{kg})$ & & & & \\
pH & 2.61 & 2.59 & 2.53 & 2.33 \\
dynamic viscosity & 1.60 & 2.13 & 3.07 & $9.53\left(40^{\circ} \mathrm{C}\right)$ \\
$\quad\left(\mathrm{mm}^{2} / \mathrm{s}, 20^{\circ} \mathrm{C}\right)$ & & & &
\end{tabular}

emission content was the average analysis results with deviation of $\pm 5 \%$.

\section{Results and Discussion}

3.1. Physical Properties of the Aqueous Bio-oil Fraction and the Formula Emulsifier. Although less complex than pure biooil, the three aqueous extracts still contain the chemical compositions of over 100 compounds; most of them are concentrated ketene, aldehydes, and acetic acid. Aromatic compounds such as 2-methoxy-4-methyl-phenol also account for several percent. The final aqueous solution of Bal contained 19.9 wt $\%$ organics as $\mathrm{CH}_{1.18} \mathrm{O}_{0.61}$. $\mathrm{Ba} 2$ and $\mathrm{Ba} 3$, which are derived from low water addition, contained more organics (27.4 wt \% and 38.6 wt $\%$ as $\mathrm{CH}_{1.15} \mathrm{O}_{0.59}$ and $\mathrm{CH}_{1.14} \mathrm{O}_{0.56}$, respectively) and their physical properties are shown in Table 1. Because of the high water contents of $\mathrm{Ba} 1, \mathrm{Ba} 2$, and $\mathrm{Ba}$, their heating values were not analyzed.

It can be seen that the density, acidity, and dynamic viscosity of the aqueous bio-oil fraction decreased as the amount of water added increased, which is favorable for combustion. However, high water content (>60 wt \%) was disadvantageous. ${ }^{18,19}$ Thus, emulsification of the aqueous bio-oil fraction with diesel to form a W/O emulsion should be

(18) Cornelissen, T.; Jans, M.; Yperman, J.; Reggers, G.; Schreurs, S.; Carleer, R. Flash co-pyrolysis of biomass with polyhydroxybutyrate:

Part 1. Influence on bio-oil yield, water content, heating value and the production of chemicals. Fuel 2008, 87 (12), 2523-2532.

(19) Lang, X.; Dalai, A. K.; Bakhshi, N. N.; Reaney, M. J.; Hertz, P. B. Preparation and characterization of bio-diesels from various biooils. Bioresour. Technol. 2001, 80 (1), 53-62.

(20) Junming, X.; Jianchun, J.; Yunjuan, S.; Yanju, L. Bio-oil upgrading by means of ethyl ester production in reactive distillation to remove water and to improve storage and fuel characteristics. Biomass Bioenergy 2008, 32 (11), 1056-1061.

(21) Nguyen, D.; Honnery, D. Combustion of bio-oil ethanol blends at elevated pressure. Fuel 2008, 87 (2), 232-243.

(22) Xiong, W.-M.; Zhu, M.-Z.; Deng, L.; Fu, Y.; Guo, Q.-X. Esterification of Organic Acid in Bio-Oil using Acidic Ionic Liquid Catalysts. Energv Fuels 2009, 23 (4), 2278-2283.
Table 2. Physical Properties of Two Formula Emulsifiers

\begin{tabular}{lll}
\hline \multicolumn{1}{c}{ physical property } & \multicolumn{1}{c}{ Mix1 } & \multicolumn{1}{c}{ Mix2 } \\
\hline hydrophilic lipophilic balance (HLB) number & 6.6 & 7.6 \\
dynamic viscosity $\left(\mathrm{mm}^{2} / \mathrm{s}, 20^{\circ} \mathrm{C}\right)$ & 588.3 & 479.5 \\
color & light yellow & dark yellow \\
odor & pungent & pungent
\end{tabular}

Table 3. Effect of Aqueous Bio-oil Fraction:Diesel Mass Ratio on Emulsion Stability ${ }^{a}$

Stability Times (h) at Various Aqueous Bio-oil Fraction:Diesel Mass Ratios

\begin{tabular}{|c|c|c|c|c|c|c|c|c|c|}
\hline & \multicolumn{3}{|c|}{ Bal:Diesel } & \multicolumn{3}{|c|}{ Ba2:Diesel } & \multicolumn{3}{|c|}{ Ba3:Diesel } \\
\hline & $1: 9$ & $2: 8$ & $3: 7$ & $1: 9$ & $2: 8$ & $3: 7$ & $1: 9$ & $2: 8$ & $3: 7$ \\
\hline with Mixl addition & $\gg 24$ & 12 & 6 & $\gg 24$ & 6 & 1 & 6 & 1 & 1 \\
\hline with Mix 2 addition & $\gg 24$ & 8 & 1 & $\gg 24$ & 3 & 1 & 1 & 1 & 1 \\
\hline
\end{tabular}

${ }^{a}$ Conditions: mass ratio of emulsifier to aqueous bio-oil fraction/ diesel mixture, $5 \mathrm{wt} \%$; ultrasonic time, $120 \mathrm{~min}$; and ultrasonic power, $1 \mathrm{~kW}$.

adopted to fulfill the combustion requirement and usage of bio-oil in the diesel generator. ${ }^{20-22}$

The choice of the emulsifier to stabilize the ABF/D emulsion and avoid (or delay) the emulsion breaking is a procedure driven by the empirical criterion of the hydrophilic lipophilic balance (HLB) number being from 4 to 8 . Because of their efficiency in preparing emulsified diesel with water, Span, Tween, and TritonX-100 were chosen to make formula emulsifier in this paper. $n$-Octanol was used as a co-surfactant, to reduce the viscosity and adjust the HLB number to improve the time stability of the resulting emulsion. Two different formula emulsifiers were prepared, and their properties are shown in Table 2.

The formula emulsifiers of Mix 1 and Mix2 had increased HLB numbers, of 6.6 and 7.6, respectively. The hydrophilicity property, relative to decreased dynamic viscosity, was due to the addition of TritonX-100, whose HLB number is 14.5. Yet, with decreased dynamic viscosity, the color of the emulsifiers became darker with a pungent odor.

3.2. ABF/D Stratification. It is necessary to establish the ranges of process operating conditions, which are required to produce stable emulsion fuels. A series of runs was performed to determine the relationship between process conditions and emulsion stability. To obtain a long-term stable and single-phase emulsion, emulsifier amounts as high as $5 \mathrm{wt} \%$ Mix 1 or Mix 2 were added. After incubating some of the $\mathrm{ABF} / \mathrm{D}$ emulsion at room temperature, the homogeneous single phase of the emulsion became unstable and phase separation could be observed, which became stratified into two layers, because of flocculation, coalescence, or breaking. ${ }^{15,21}$ The stability time before obvious stratification is shown in Table 3.

Phase separation was not observed in Bal/diesel and $\mathrm{Ba} 2 /$ diesel emulsions after $24 \mathrm{~h}$ when the aqueous bio-oil fraction:diesel mass ratio was 1:9 with the addition of $5 \mathrm{wt} \%$ Mix 1 or Mix2, while the stability time was reduced to $<12 \mathrm{~h}$ when the mass ratio increased to $2: 8$ or $3: 7$. The $\mathrm{Ba} 3 /$ diesel emulsions became stratified within $<6 \mathrm{~h}$. It could be concluded that the stability of $\mathrm{ABF} / \mathrm{D}$ emulsions increased as the water:bio-oil ratio increased and the aqueous bio-oil fraction addition decreased. Obviously, the nonstratification time with the addition of Mix 1 was longer than that of Mix2, where no TritonX-100 surfactant was added. 
Table 4. Effect of Mass Ratio of Aqueous Bio-oil Fraction to Diesel on ABF/D Stratification Rates

Stratification Rates of ABF/D Emulsions (vol \%) at Various Aqueous Bio-oil Fraction:Diesel Mass Ratios ${ }^{a}$

Ba1:Diesel Ba2:Diesel Ba3:Diesel

$\begin{array}{llllllllll}\text { with Mixl addition } & 0 & 4 & 16 & 0 & 4 & 24 & 8 & 24 & 32\end{array}$

$\begin{array}{llllllllll}\text { with Mix2 addition } & 0 & 8 & 23 & 0 & 12 & 32 & 12 & 28 & 36\end{array}$

${ }^{a}$ Conditions: mass ratio of emulsifier to aqueous bio-oil fraction/ diesel mixture: $5 \mathrm{wt} \%$; ultrasonic time, $120 \mathrm{~min}$; ultrasonic power, $1 \mathrm{~kW}$.

The main reason might be distant arrangement of lipophilic groups of isooctylphenyl from TritonX-100, relative to monocarboxylic ester groups from the Tween or Span surfactant. ${ }^{23}$ Therefore, the interface of $\mathrm{W} / \mathrm{O}$ droplets in the Mix2 emulsified liquid was not strong enough to sustain the collision due to diffusion during incubation and aging, where the single and dispersed droplets became swollen micelles. ${ }^{24}$ When its dimension reached a critical value, the phase separation had occurred.

For those emulsions prepared from a high aqueous bio-oil fraction:diesel mass ratio, the stratification rate increased as time elapsed. Reliable rates were recorded after incubating for 30 days at room temperature, as listed in Table 4. All Mix1-added emulsions exhibited lower rates and better stability than Mix2-added emulsions. For example, the stratification rate for Mix 1-emulsified Ba2/diesel (mass ratio $=2: 8$ ) was $4 \mathrm{vol} \%$, which was better than $12 \%$ of the Mix2added emulsions, because of the different lipophilic groups of TritonX-100 mentioned previously.

Apparently, at the same aqueous bio-oil fraction:diesel ratio, Ba3/diesel emulsions showed higher stratification rates than $\mathrm{Ba} 2 /$ diesel or $\mathrm{Ba} 1 /$ diesel emulsions, resulting from the relatively large density difference between the dispersed aqueous bio-oil fraction and continuous diesel phase in Table 1. In addition, the high content of water-soluble carbohydrates, such as $\mathrm{CH}_{1.14} \mathrm{O}_{0.56}$ of $\mathrm{Ba} 3$, and the lower water content in the $\mathrm{Ba} 3$ may be another important reason for stratification, because of the decreased critical micelle concentration $(\mathrm{CMC})$ and less solubilization ability of these organic compounds in the inner phase of $\mathrm{W} / \mathrm{O}$ droplets, especially for those slightly soluble aromatic compounds (as phenol), which may become insoluble. Moreover, the droplets sank to the bottom, which caused phase separation, and the dense lower layer was mainly caused by the flocculation or coalescence of these compounds in water.

At the same time, the increase in the aqueous bio-oil fraction:diesel mass ratio has a negative effect on $\mathrm{ABF} / \mathrm{D}$ stability, as shown by the increasing stratification ratios of 0 vol $\%, 4 \mathrm{vol} \%$, and $16 \mathrm{vol} \%$ when the Ba1:diesel ratios increased as 1:9, 2:8, and 3:7, respectively, under Mix 1. The decreased content of aqueous bio-oil phase in the emulsion, which was dispersed into smaller micelle droplets in larger amounts of continuous diesel phase under the emulsifier and were not likely to flocculate, may be the main reason. Even after 30 days, Ba1/diesel and Ba2/diesel emulsions at an

(23) Xu, Q. Y.; Nakajima, M.; Nabetani, H.; Ichikawa, S.; Liu, X. Q. Effect of the dispersion behavior of a nonionic surfactant on surface activity and emulsion stability. J. Colloid Interface Sci. 2001, 242 (2), $443-449$

(24) Fratini, E.; Bonini, M.; Oasmaa, A.; Solantausta, Y.; Teixeira, J.; Baglioni, P. SANS Analysis of the Microstructural Evolution during the Aging of Pyrolysis Oils from Biomass. Langmuir 2005, 22 (1), 306-312.
Table 5. Effect of Emulsifier Amount on ABF/D Stratification Rates

Stratification Rates of ABF/D Emulsions $(\mathrm{vol} \%)$ at Various Emulsifier Amounts ${ }^{a}$

\begin{tabular}{|c|c|c|c|c|c|c|}
\hline & \multicolumn{3}{|c|}{ Emulsifier: Ba1/diesel } & \multicolumn{3}{|c|}{ Emulsifier: Ba2/diesel } \\
\hline & $1 \mathrm{wt} \%$ & $3 \mathrm{wt} \%$ & $5 \mathrm{wt} \%$ & $1 \mathrm{wt} \%$ & $3 \mathrm{wt} \%$ & $5 \mathrm{wt} \%$ \\
\hline & 8 & 0 & 0 & 12 & 4 & 0 \\
\hline with Mix2 addition & 12 & 0 & 0 & 16 & 6 & 0 \\
\hline
\end{tabular}

${ }^{a}$ Conditions: mass ratio of aqueous bio-oil fraction to diesel, 1:9; ultrasonic time, $120 \mathrm{~min}$; and ultrasonic power, $1 \mathrm{~kW}$.

Table 6. Effect of Ultrasonic Time on Stratification Rates of Ba1/diesel Emulsions

Stratification Rates of Ba1/Diesel Emulsions $(\mathrm{vol} \%)$ at Various Ultrasonic Times ${ }^{a}$

\begin{tabular}{lccc} 
& $60 \mathrm{~min}$ & $90 \mathrm{~min}$ & $120 \mathrm{~min}$ \\
\cline { 2 - 4 } with Mix 1 addition & 7 & 4 & 0 \\
with Mix2 addition & 10 & 6 & 0
\end{tabular}

${ }^{a}$ Conditions: mass ratio of Ba1 to diesel, 1/9; mass ratio of emulsifier to aqueous bio-oil fraction/diesel mixture, $3 \mathrm{wt} \%$; and ultrasonic power, $1 \mathrm{~kW}$.

aqueous bio-oil:diesel mass ratio of 1:9 did not stratify, indicating excellent emulsification performance. Therefore, $\mathrm{Ba} 1 /$ diesel and $\mathrm{Ba} 2 /$ diesel emulsions were chosen for the following experiments.

The effect of emulsifier amount on stratification rate after 30 days is listed in Table 5. It shows that the stability of the $\mathrm{Ba} 1 /$ diesel or Ba2/diesel emulsion increased as the Mix 1 or Mix 2 amount increased from $1 \mathrm{wt} \%$ to $5 \mathrm{wt} \%$, because only an adequate amount of emulsifier could be able to form a closely adsorbed monolayer interface onto the $\mathrm{ABF} / \mathrm{D}$ droplets with enough mechanical strength to avoid droplets coalescence and stratification. ${ }^{25,26}$ On the other hand, excess emulsifier micelles under high addition amounts will adsorb onto the surface of the emulsion droplets, also leading to coalescence. ${ }^{27}$ Obviously, 3 wt \% Mix 1 (or Mix2) addition was sufficient to form stable Ba1/diesel emulsion and high emulsifier addition of $5 \mathrm{wt} \%$ did not exceed the emulsion stability limit and cause stratification of $\mathrm{Ba} 1 /$ diesel emulsion. The single phase of $\mathrm{Ba} 2 /$ diesel emulsion remained stable only when the emulsifier amount was increased to $5 \mathrm{wt} \%$. It might be due to the relatively higher organics content of 27.4 wt $\%$ as $\mathrm{CH}_{1.15} \mathrm{O}_{0.59}$ for $\mathrm{Ba} 2$, compared with the organics content of 19.9 wt $\%$ as $\mathrm{CH}_{1.18} \mathrm{O}_{0.61}$ for $\mathrm{Ba} 1$ in section 3.1. Most of the increased organics of $\mathrm{Ba} 2$ were slightly soluble aromatic compounds (phenols, guaiacols, syringols, and mono or oligosaccharides), which had low solubilization ability in the $\mathrm{W} / \mathrm{O}$ droplets and required more hydrophilic polyoxyethylene groups to surround and form an amphiphilic molecular interface, to stabilize the $\mathrm{W} / \mathrm{O}$ droplets of the dispersed organics phase in the emulsion. ${ }^{28}$

(25) Song, M. G.; Jho, S. H.; Kim, J. Y.; Kim, J. D. Rapid evaluation of water-in-oil (w/o) emulsion stability by turbidity ratio measurements. J. Colloid Interface Sci. 2000, 230 (1), 213-215.

(26) Ikura, M.; Mirmiran, S.; Stanciulescu, M.; Sawatzky, H. Pyrolysis liquid-in-diesel oil microemulsions, U.S. Patent 5,820,640, Oct. 13, 1998.

(27) Juang, R. S.; Lin, K. H. Ultrasound-assisted production of W/O emulsions in liquid surfactant membrane processes. Colloids Surf. A 2004, $238(1-3), 43-49$

(28) Ikura, M.; Stanciulescu, M.; Hogan, E. Emulsification of pyrolysis derived bio-oil in diesel fuel. Biomass Bioenergy 2003, 24 (3), 221232. 
Table 7. Emulsification Influence Factor Analysis on ABF/D Stability

\begin{tabular}{|c|c|c|c|c|c|}
\hline & \multicolumn{5}{|c|}{ Influence Factor Index } \\
\hline & $\begin{array}{l}\text { aqueous bio-oil } \\
\text { fraction } \mathrm{Ba}\end{array}$ & $\begin{array}{c}\text { aqueous bio-oil fraction: } \\
\text { diesel mass ratio }\end{array}$ & emulsifier type & emulsifier amount & ultrasonic time \\
\hline$\overline{\text { expected index }}$ & 1 & 1 & 1 & 1 & 1 \\
\hline actual index & 0.92 & 0.84 & 1 & 0.92 & 0.93 \\
\hline index influence $^{a}$ & 0.08 & 0.15 & 0 & 0.06 & 0.05 \\
\hline
\end{tabular}

Table 8. Physical Properties of ABF/D Emulsion

\begin{tabular}{|c|c|c|c|c|c|c|c|}
\hline \multirow[b]{3}{*}{ physical property } & \multirow[b]{3}{*}{ 0\# diesel } & \multicolumn{6}{|c|}{ Value for Various ABF/D Emulsions } \\
\hline & & \multicolumn{2}{|c|}{$\begin{array}{c}\text { 3:10:90 Emulsifer:Ba: } \\
\text { Diesel Ratio (wt \% basis) }\end{array}$} & \multicolumn{4}{|c|}{$\begin{array}{c}\text { 5:10:90 Emulsifer:Ba: } \\
\text { Diesel Ratio (wt\% basis) }\end{array}$} \\
\hline & & $\begin{array}{c}\text { Mix 1:Ba1: } \\
\text { diesel }\end{array}$ & $\begin{array}{c}\text { Mix2:Ba1: } \\
\text { diesel }\end{array}$ & $\begin{array}{c}\text { Mix l:Ba1: } \\
\text { diesel }\end{array}$ & $\begin{array}{c}\text { Mix2:Ba1: } \\
\text { diesel }\end{array}$ & $\begin{array}{c}\text { Mix 1:Ba2: } \\
\text { diesel }\end{array}$ & $\begin{array}{c}\text { Mix2:Ba2: } \\
\text { diesel }\end{array}$ \\
\hline water content (wt \%) & & 1.39 & 0.47 & 0.67 & 2.96 & 1.08 & 1.05 \\
\hline density $(\mathrm{g} / \mathrm{mL})$ & 0.860 & 0.888 & 0.882 & 0.890 & 0.888 & 0.892 & 0.892 \\
\hline LHV (MJ/kg) & 45.0 & 44.25 & 43.94 & 44.03 & 43.40 & 43.58 & 44.19 \\
\hline $\mathrm{pH}$ & 5.26 & 2.95 & 2.90 & 3.0 & 2.8 & 2.69 & 2.68 \\
\hline dynamic viscosity & 4.58 & 6.34 & 6.45 & 6.50 & 8.30 & 6.57 & 7.49 \\
\hline
\end{tabular}

Effect of ultrasonic time on Ba1/diesel emulsion stability with $3 \mathrm{wt} \%$ emulsifier is listed in Table 6 . As the ultrasonic time increased from $60 \mathrm{~min}$, to $90 \mathrm{~min}$, to $120 \mathrm{~min}$, the stratification rates decreased and the transparent emulsion formed as the color changed from milky to dark gray gradually. For Mix1- or Mix2-emulsified Ba1/diesel emulsions, the liquid could remain stable for more than 30 days at room temperature after an ultrasonic time of $120 \mathrm{~min}$.

3.3. Emulsification Influence Factor Analysis on ABF/D Stability. In this paper, the main factors that affect emulsification performance are aqueous bio-oil fraction $\mathrm{Ba}$, aqueous bio-oil fraction:diesel mass ratio, type and amount of emulsifier, and ultrasonic time. The results, using a simple factor analysis approach method, are listed in Table 7. The stable Ba1/diesel emulsion (mass ratio $=1: 9,5$ wt $\%$ Mix 1 , ultrasonic time $=120 \mathrm{~min}$ ) without stratification is identified as the expected target for excellent emulsification. For the above five influencing factors, the expected indexes were all defined as 1. The actual index for each factor was the maximum derivation of the practical emulsification result from the expected ones when the other four factors were under their expected conditions.

For example, there are three levels of aqueous bio-oil fraction ( $\mathrm{Ba} 1, \mathrm{Ba} 2$, and $\mathrm{Ba} 3)$, and the stratification rates were $0 \mathrm{vol} \%, 0 \mathrm{vol} \%$, and $8 \mathrm{vol} \%$, respectively, at a aqueous bio-oil fraction:diesel mass ratio of $1: 9$, with a $5 \mathrm{wt} \%$ Mix 1 addition and an ultrasonic time of $120 \mathrm{~min}$ (see Table 4 in section 3.2). Therefore, the maximum derivation of the $\mathrm{Ba}$ factor was 0.92 , which was defined as the actual index. The actual indexes of the other four factors were obtained by following the same definition. The index influence was calculated following factor analysis approach principles as the footnotes in Table 7 show, without considering the correlation between factors. $^{29}$ The index influence of $\mathrm{Ba}$ was the multiplication product of the maximum index derivation of $\mathrm{Ba}$ and the expected indexes of the other four factors. In addition, for the index influence calculation of the

(29) Song, F.; Gao, Y. Chemical characteristics of precipitation at metropolitan Newark in the U.S. East Coast. Atmos. Environ. 2009, 43 (32), 4903-4913. following four factors, the expected indexes of the forgoing factor were substituted by the actual indexes in the equations. The results indicate that the aqueous bio-oil fraction: diesel mass ratio is the most important factor that affects the emulsion stability in this study.

3.4. Physical Properties of ABF/D Emulsion. The six emulsions without stratification in Table 5 were stable and opaque with no evidence of phase separation, which had a higher level of stability than conventional bio-oils and is promising in diesel generator applications.

Further, physical properties of the emulsions were more similar to diesel than that of bio-oil itself, with regard to water content, density, and LHV. However, the $\mathrm{pH}$ and dynamic viscosity values were still high, because of the carbohydrates (such as alcohols, phenols, aldehydes, ketones, acids, and esters) solubilizing in the $\mathrm{W} / \mathrm{O}$ droplets of the emulsions. Moreover, the high content of water-soluble organics in $\mathrm{Ba} 2$ might be the main reason for low $\mathrm{pH}$ values of $\mathrm{Ba} 2 /$ diesel emulsions. The higher dynamic viscosity of Mix2-added emulsions, compared with Mix1-added emulsions, may be the result of weaker emulsification and disruption ability of aqueous bio-oil fraction droplets by Mix2, because of the structure of iso-octylphenyl groups of TritonX-100, which were 6.50 and 8.30 for Mix1/Ba1/diesel and Mix1/Ba1/diesel emulsions, respectively (5:10:90). High emulsifier content increased the emulsion stability in section 3.2 , but it slightly increased the dynamic viscosity in Table 8 , which resulted from the high viscosity of the emulsifier and strong interface of the $\mathrm{W} / \mathrm{O}$ droplets, which indicates the difficulties in producing the proper emulsions for engine fuels.

3.5. Preliminary Analysis of ABF/D Emulsification Mechanism. The ABF/D stratification and stability performance could only be explained by an emulsification mechanism. When bio-oil was mixed with water, most unsolvable carbon particles and lignin-derived organic compounds were sedimentated into the lower layer of the liquid, and then discarded, while the hydrophilic oxygenates $(-\mathrm{OH},-\mathrm{CHO}$, $-\mathrm{CO},-\mathrm{COOH}$ ) (including low carbon-chain carbohydrates in raw bio-oil) were concentrated in water fraction. At the same time, some compounds (such as phenol or ethyl 
acetate), which had comparably hydrophilic and hydrophobic groups and are slightly soluble in water, were also concentrated in aqueous bio-oil fraction, because of the large amount of water added into the pure bio-oil. ${ }^{20}$

When Mix1 or Mix2 emulsifier was added to $\mathrm{ABF} / \mathrm{D}$ mixtures, the hydrophilic polyoxyethylene groups (polar) had a tendency to remain in the aqueous bio-oil fraction, while the hydrophobic hydrocarbon groups (nonpolar) would orient themselves with the continuous diesel phase. ${ }^{23}$ According to this tendency, the emulsifier molecules were enriched from the bulk phase to the surface or interface of the aqueous bio-oil fraction and diesel, thus oriented/ordered arrangements of $\mathrm{W} / \mathrm{O}$ emulsions were formed and the surface tension was decreased, where the hydrophobic carbohydrates-concentrated aqueous fraction was concentrated to form single molecular membrane on the emulsifier surface at low amounts of added emulsifier. When the emulsifier amount is higher than the CMC, the micelles were formed with no further decrease in surface tension. Moreover, the slightly soluble compounds as phenols or esters was solubilized in the formed micelles, which resulted in relatively stable homogeneous emulsions. ${ }^{7}$

Therefore, it could be concluded that the emulsification mechanism of the $\mathrm{ABF} / \mathrm{D}$ emulsion was that the polar compounds in the aqueous bio-oil fraction (such as water and oxygenates (ketones, acids, aldehydes, alcohols)) were embodied in the droplets of $\mathrm{W} / \mathrm{O}$ emulsion stably, while small amount of esters, and aromatic hydrocarbons (phenols), were solubilized in the hydrophilic micelles of the polyoxyethylene fraction. ${ }^{30}$ However, when the high carbohydrate organics content of $\mathrm{Ba} 3$-contained $\mathrm{W} / \mathrm{O}$ droplets was in the emulsion, its solubilization ability could be reduced, especially under TritonX-100-added Mix2 emulsifier due to the distant arrangement of amphiphilic groups and caused high stratification rate.

3.6. Analysis of Ultrasonic Action on ABF/D Emulsification. The structural water and oxygenates in the aqueous bio-oil fraction makes it immiscible with hydrocarbon-based diesel oil. To emulsify this thermodynamically unstable system, energy is needed to disperse the insoluble aqueous bio-oil phase (dispersed phase) in the form of small droplets in the diesel phase (continuous phase).

Ultrasound is an additional, efficient method to dissipate mechanical energy, which is required for droplet disruption, in a liquid. Moreover, the use of ultrasonic power has been proven to produce smaller droplet particle sizes at lower emulsifier levels, not requiring any external agitation, than mechanical agitation treatment under the same conditions, which makes it suitable for diesel emulsification and apply widely on a laboratory scale. ${ }^{31}$ For comparison, a Ba1/diesel emulsion (Mix1:Ba1:diesel = 3:10:90) was also prepared under strong mechanical agitation, which stratified after 10 days with less stability time than those under ultrasonic emulsification.

There are several possible mechanisms of droplet formation and disruption under the effect of ultrasound. Cavitation is the mechanism generally regarded as crucial. Imploding cavitation bubbles in the vicinity of transducer

(30) Kim, Y. H.; Wasan, D. T.; Breen, P. J. A study of dynamic interfacial mechanisms for demulsification of water-in-oil emulsions. Colloids Surf. A 1995, 95 (2-3), 235-247.

(31) Behrend, O.; Ax, K.; Schubert, H. Influence of continuous phase viscosity on emulsification by ultrasound. Ultrason. Sonochem. 2000, 7 (2), 77-85. radiating surface causes intensive shock waves in the surrounding liquid and liquid jets formation. ${ }^{32}$ This may bring the rough premix $\mathrm{ABF} / \mathrm{D}$ droplet disruption in the vicinal dispersion zone. In a second step, the newly formed droplets must be stabilized against coalescence. For this purpose, Mix1 or Mix2 added to the system was adsorbed at the interface between two phases, because of their amphiphilic molecular structure, and thereby stabilized the droplets of the dispersed phase of the emulsion. Parameters such as the emulsifier and the viscosity of the dispersed and continuous phase positively affect cavitation in liquids. Generally speaking, the smaller droplet size of the dispersed phase after phase disruption may improve emulsification performance with higher emulsion stability under ultrasound treatment.

The entropy increase during ultrasonic emulsification on the $\mathrm{ABF} / \mathrm{D}$ emulsion is given as

$$
\mathrm{d} S_{1}=\frac{\delta Q_{1}}{T}+\delta S_{\mathrm{g} 1}
$$

where $\delta Q_{1}$ is the amount of heat absorbed by the $\mathrm{ABF} / \mathrm{D}$ emulsion $\left(\delta Q_{1}>0\right)$ and $\delta S_{\mathrm{g} 1}$ is the entropy increase from chemical reaction during ultrasonic treatment $\left(\delta S_{\mathrm{g} 1}>0\right) .{ }^{33}$ The entropy increase during the incubating period after ultrasonic action is given as

$$
\mathrm{d} S_{2}=\frac{\delta Q_{2}}{T}+\delta S_{\mathrm{g} 2}
$$

where $\delta Q_{2}$ is the exothermic heat due to temperature decreasing $\left(\delta Q_{2}<0\right)$ and $\delta S_{\mathrm{g} 2}$ is the entropy increase from chemical reaction during incubation $\left(\delta S_{\mathrm{g} 2}>0\right) .{ }^{13}$ Among them, one part of the input energy from an ultrasonic transducer is converted to heat for the $\mathrm{ABF} / \mathrm{D}$ emulsion as the $\mathrm{ABF} / \mathrm{D}$ emulsion temperature increased; the other part became the chemical energy of the reactions. Because of the same temperature change during ultrasonic action and incubation, $\left|\delta Q_{1}\right|=\left|\delta Q_{2}\right|$. Thus, the entire entropy increase during ultrasonic emulsification and incubation at room temperature is given as

$$
\begin{aligned}
\mathrm{d} S_{\mathrm{u}} & =\mathrm{d} S_{1}+\mathrm{d} S_{2}=\frac{\delta Q_{1}}{T}+\delta S_{\mathrm{g} 1}+\frac{\delta \mathrm{Q}_{2}}{T}+\delta S_{\mathrm{g} 2} \\
& =\delta S_{\mathrm{g} 1}+\delta S_{\mathrm{g} 2}>0
\end{aligned}
$$

whereas, for the emulsion without ultrasound, the entropy increase from chemical reaction during aging is given as

$$
\mathrm{d} S_{\mathrm{p}}=\delta S_{\mathrm{g}}>0
$$

Moreover, because of the cavitation of ultrasonic treatment and energy converted from the transducer to chemical energy, $\mathrm{d} S_{\mathrm{u}}=\delta S_{\mathrm{g} 1}+\delta S_{\mathrm{g} 2}>\mathrm{d} S_{\mathrm{p}}$, which indicated that the ultrasonic emulsification had a larger entropy increase than that without ultrasonics. That accelerated the conversion of the $\mathrm{ABF} / \mathrm{D}$ emulsion to the equilibrium state and stabilized the emulsion. ${ }^{7}$

3.7. Thermogravimetric Analysis of Diesel, Bio-oil, and ABF/D emulsion. Although some of stratified layers can be

(32) Moussatov, A.; Granger, C.; Dubus, B. Cone-like bubble formation in ultrasonic cavitation field. Ultrason. Sonochem. 2003, 10 (4-5), 191-195

(33) Das, A. K.; Jha, B. L. Ultrasonic study of binary mixtures of some monohydroxy alcohols, ketones, and nitrobenzene with DMSO as common solvent. J. Mol. Liq. 1994, 59 (2-3), 161-171. 


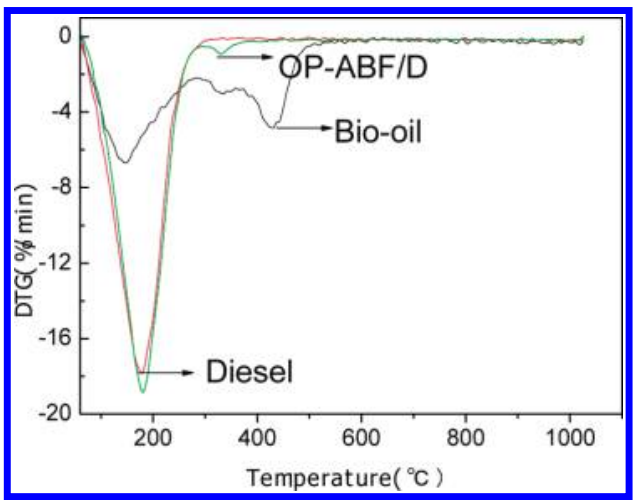

Figure 2. DTG curves of bio-oil, diesel, and the ABF/D emulsion.

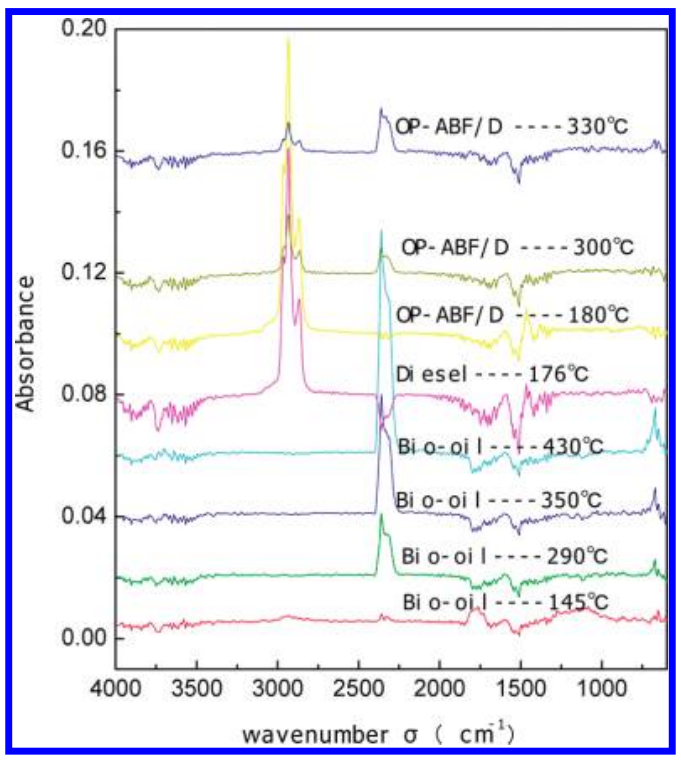

Figure 3. Online FTIR spectra of thermal analysis tailgas of bio-oil, diesel, and the OP-ABF/D emulsion.

redispersed into the emulsion by gentle shaking, their presence is undesirable in fuel supply. Compared to the other five $\mathrm{ABF} / \mathrm{D}$ emulsions, a stable Mix 1/Ba1/diesel (3:10:90) emulsion showed relatively high emulsification performance. In addition, because its viscosity increment was only $38.4 \%$ with $\mathrm{pH}$ decreasing from 5.26 to 2.95 by pure diesel, it was identified as the optimized AFB/D emulsion, denoted as the OP-ABF/D emulsion, and used in the following combustion experiments. A high amount of Mix 1 emulsifier (3 wt \%) was added because the objective of these studies was to investigate the combustion properties of stable $\mathrm{ABF} / \mathrm{D}$ emulsions feeding into an engine, without the influence of coalescence and stratification.

The largest weight loss peak at $145^{\circ} \mathrm{C}$ of pure bio-oil at $60-220{ }^{\circ} \mathrm{C}$ in Figure 2 was attributed to the light volatile fraction (especially water, ketone, aldehydes, formic acid, and alcohols), as confirmed by the online Fourier transform infrared (FTIR) spectra of the tailgas (see Figure 3, which indicates a $\mathrm{C}=\mathrm{O}$ band at a wavenumber $(\sigma)$ of $1765 \mathrm{~cm}^{-1}$ of carboxylic acid, ${ }^{11} \mathrm{a} \mathrm{O}-\mathrm{H}$ band at $1279 \mathrm{~cm}^{-1}$ of alcohols, and a $\mathrm{C}-\mathrm{O}$ band at $1104 \mathrm{~cm}^{-1}$ of alcohol or ether in the bio-oil

(34) Li, C.; Suzuki, K. Kinetics of Perovskite Catalyzed Biomass Tar Combustion Studied by Thermogravimetry and Differential Thermal Analysis. Energv Fuels 2009, 23 (5), 2364-2369.

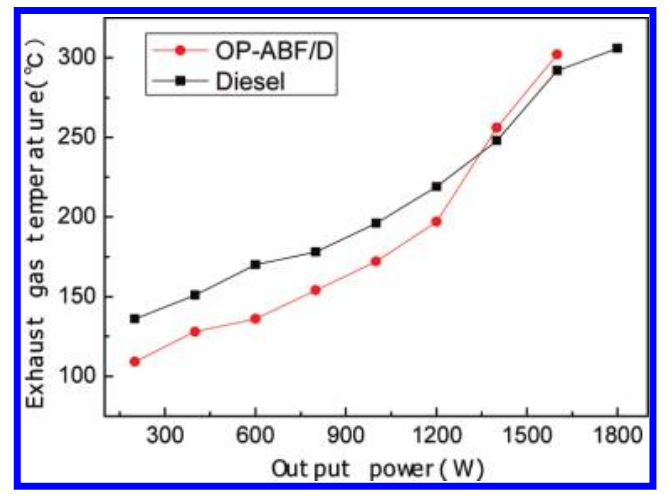

Figure 4. Effect of generator output power on exhaust gas temperature.

spectra at $145^{\circ} \mathrm{C} .{ }^{34,35}$ In the same time, the adsorption band at $2361 \mathrm{~cm}^{-1}$, which corresponds to $\mathrm{CO}_{2}$, was also distinguishable at $145{ }^{\circ} \mathrm{C}$, which may have resulted from the combustion of these volatile compounds. With increasing temperature, $\mathrm{CO}_{2}$ became the main component in the tailgas after bio-oil thermogravimetric analysis, as shown by the increasing intensity at $2361 \mathrm{~cm}^{-1}$ from $290^{\circ} \mathrm{C}$ to $430{ }^{\circ} \mathrm{C}$, and corresponds to the oxidative cracking and combustion of heavy fraction of bio-oil. It also implied quite different volatility and combustion characteristics of light fraction and heavy fraction of pure bio-oil. Therefore, it is quite necessary to separate bio-oil into different fractions to make full of use them.

The DTG curve of the OP-ABF/D emulsion shows a weight loss peak similar to that of diesel, which is observed at 180 and $176{ }^{\circ} \mathrm{C}$, respectively, because of the relative low bio-oil content and dilution by diesel in the emulsion. According to the most intensive adsorption band at $2933 \mathrm{~cm}^{-1}$ in the FTIR spectra, this weight loss was attributed to the thermal decomposition and evaporation of hydrocarbons, which was the symmetric $\mathrm{C}-\mathrm{H}$ stretches of $-\mathrm{CH}_{3}$ groups from the pyrolyzed compounds in the tailgas of the OP-ABF/D emulsion or diesel. ${ }^{36}$ There was no $\mathrm{CO}_{2}$ produced during the entire thermogravimetric analysis range at $40-1100{ }^{\circ} \mathrm{C}$ of diesel. Yet, the DTG curve of the OP-ABF/ $\mathrm{D}$ tailgas indicated that a small amount of $\mathrm{CO}_{2}$ was produced after $300{ }^{\circ} \mathrm{C},{ }^{37}$ which should result from the low content of heavy-fraction combustion from the aqueous bio-oil fraction in the emulsion, which was less-volatile according to the weak weight loss peak at $330^{\circ} \mathrm{C}$ in Figure 2. ${ }^{11,38}$ The weight loss at $330{ }^{\circ} \mathrm{C}$ of the OP-ABF/D emulsion also implied the presence of relatively complex components and worse combustion properties of emulsion in the diesel generator below, although the content of those components may be less, compared to the main weight loss peak at $\sim 180^{\circ} \mathrm{C}$.

3.8. Combustion Properties of Diesel, Bio-oil, and the OPABF/D Emulsion in a Diesel Generator. Figure 4 shows the

(35) Das, P.; Sreelatha, T.; Ganesh, A. Bio oil from pyrolysis of cashew nut shell-characterisation and related properties. Biomass Bioenergy 2004, 27 (3), 265-275.

(36) ǒZbay, N.; Uzun, B. B.; Varol, E. A.; Pǔtǔn, A. E. Comparative analysis of pyrolysis oils and its subfractions under different atmospheric conditions. Fuel Process. Technol. 2006, 87 (11), 1013-1019.

(37) Li, S.; Lyons-Hart, J.; Banyasz, J.; Shafer, K. Real-time evolved gas analysis by FTIR method: an experimental study of cellulose pyrolysis. Fuel 2001, 80 (12), 1809-1817.

(38) Guo, X.; Zheng, Y.; Zhang, B.; Chen, J. Analysis of coke precursor on catalyst and study on regeneration of catalyst in upgrading of bio-oil. Biomass Bioenergy 2009, 33 (10), 1469-1473. 

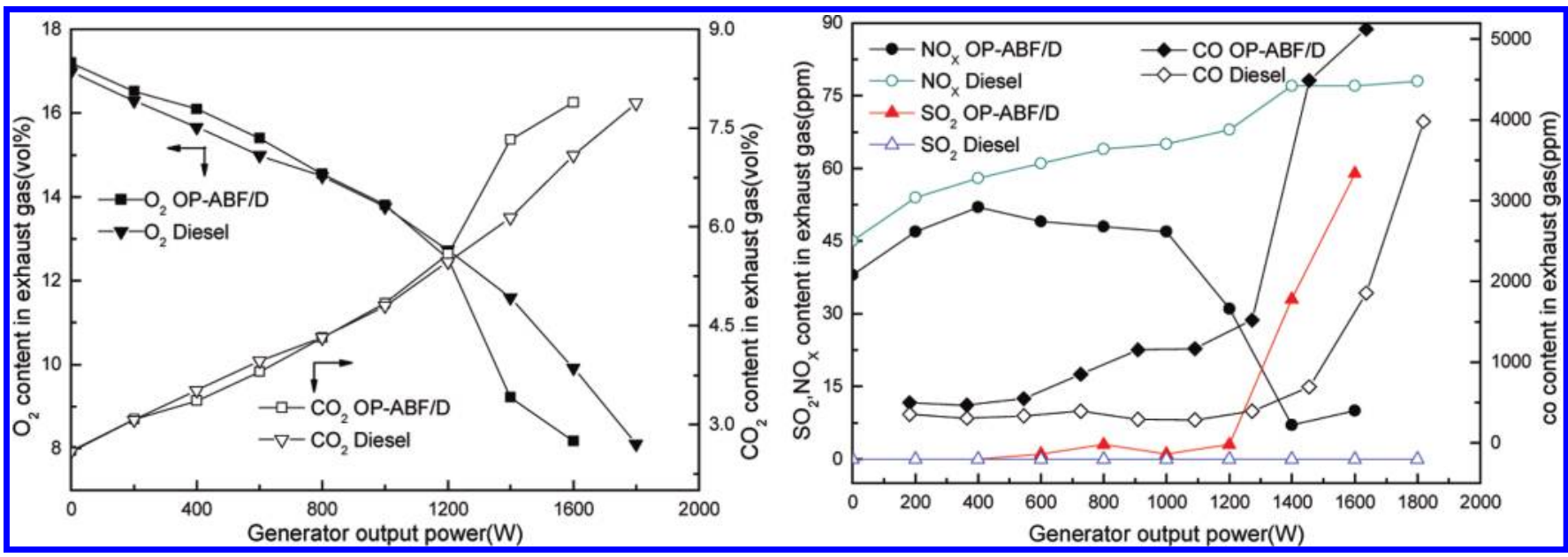

Figure 5. Effect of generator output power on the $\mathrm{O}_{2}, \mathrm{CO}_{2}, \mathrm{SO}_{2}, \mathrm{NO}_{\mathrm{X}}$, and $\mathrm{CO}$ contents in the exhaust gas.

tendency for the exhaust gas temperature to increase with the increased generator output power, using the $\mathrm{OP}-\mathrm{ABF} / \mathrm{D}$ emulsion or diesel as feedstock. When the output power is $<1200 \mathrm{~W}$, the exhaust gas temperature with the OP-ABF/D feed was lower than that observed using diesel, which may have resulted from the emulsion droplet microexplosion by water vaporization during the $\mathrm{OP}-\mathrm{ABF} / \mathrm{D}$ emulsion. First, the water phase wrapped in the $\mathrm{W} / \mathrm{O}$ droplets underwent strong swelling phenomena when the emulsion was injected from a relatively high-pressure nozzle chamber to a lowpressure combustor, which caused sudden vaporization. Thus, the interface of $\mathrm{W} / \mathrm{O}$ droplets was collapsed and caused secondary atomization, which generates smaller liquid droplets with beneficial effects on droplet combustion and cenosphere emission. In addition, $\mathrm{OH}$ radicals provided by the water phase in the gaseous phase would inhibit the formation of soot and also increase the combustion of carbon particles. On this basis, most of the combustion was completed in the combustor and reduced the exhaust gas temperature when the OP-ABF/D emulsion was used as feedstock. Yet, because of the low injection feeding amount and the corresponding low water phase content at $<1200 \mathrm{~W}$, the resulting microexplosion intensity may be weak with similar $\mathrm{CO}_{2}, \mathrm{O}_{2}$, and $\mathrm{SO}_{2}$ content in the exhaust gas in Figure 5. According to the higher $\mathrm{CO}_{2}$ and lower $\mathrm{O}_{2}$ content in the OP-ABF/D exhaust gas than diesel in Figure 5 at $>1200 \mathrm{~W}$, microexplosion should have occurred intensively, which accelerated the $\mathrm{O}_{2}$ consumption and $\mathrm{CO}_{2}$ production during $\mathrm{OP}-\mathrm{ABF} / \mathrm{D}$ combustion. When the output power was $1600 \mathrm{~W}$ with a large amount of $\mathrm{OP}-\mathrm{ABF} / \mathrm{D}$ feed, the $\mathrm{O}_{2}$ content decreased to $8.18 \mathrm{vol} \%$ and the $\mathrm{CO}_{2}$ content increased to $7.89 \mathrm{vol} \%$, which were, respectively, $\sim 21 \%$ lower and $\sim 13.3 \%$ higher than the results obtained using diesel. However, the exhaust gas temperature of the OP-ABF/D emulsion being slightly higher than that of diesel probably resulted from the low quality of injection, because of the relatively high viscosity of the emulsion and the delayed combustion of the less-volatile compounds (as shown by the thermogravimetric analysis in section 3.7 ) in the combustor, which reduced the microexplosion effect and combustion may still occur out of the combustor with increased exhaust gas temperature.

The $\mathrm{NO}_{\mathrm{X}}$ content of the OP-ABF/D feedstock was $\sim 30 \%$ lower than that of diesel subjected to an outlet power of $<1200 \mathrm{~W}$, which decreased to $\sim 10 \mathrm{ppm}$ at $1600 \mathrm{~W}$ with a

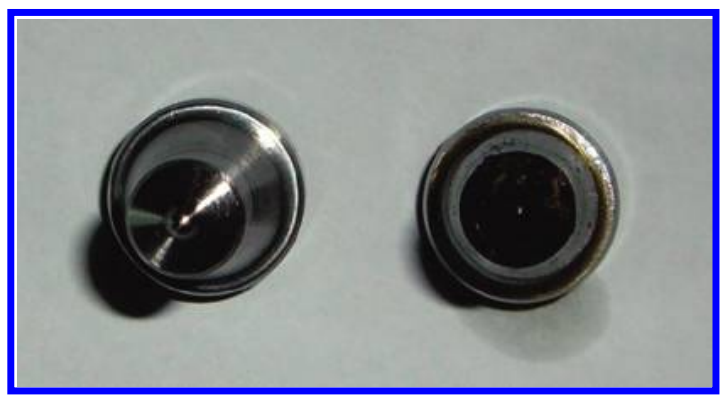

Figure 6. Comparison of injector corrosion using (left) a diesel feed and (right) the $\mathrm{OP}-\mathrm{ABF} / \mathrm{D}$ emulsion.

$87.2 \%$ decrement for diesel feedstock. Because $\mathrm{NO}_{\mathrm{X}}$ could be produced only at high temperature and under $\mathrm{O}_{2}$-rich conditions, ${ }^{39,40}$ the low $\mathrm{NO}_{\mathrm{X}}$ content in the OP-ABF/D exhaust gas indicated a lower combustor temperature in the combustor than in the diesel feed, where microexplosion may occur with high $\mathrm{O}_{2}$ consumption and some combustion heat is probably absorbed by water vaporization, especially when the output power was $>1200 \mathrm{~W}$ with a low OP-ABF/D feed amount.

Almost no $\mathrm{SO}_{2}$ was detected in the OP-ABF/D emulsion or diesel exhaust gas at $<1200 \mathrm{~W}$; the content of this compound increased quickly to $59 \mathrm{ppm}$ for the OP-ABF/D emulsion at $1600 \mathrm{~W}$, because of the increased emulsion feed amount and low sulfur content derived from bio-oil in section 2.2.41

According to the microexplosion, the vaporized water should have accelerated the $\mathrm{CO}$ combustion and decreased the $\mathrm{CO}$ content in the OP-ABF/D exhaust gas. ${ }^{42}$ Here, the $\mathrm{CO}$ content with the OP-ABF/D feed was $\sim 1.5$-fold higher than that from diesel when the output power was $<1200 \mathrm{~W}$, which increased dramatically as the output power increased to $>1200 \mathrm{~W}$ and was $5124 \mathrm{ppm}$ at $1600 \mathrm{~W}$ (1857 ppm for

(39) Shihadeh, A.; Hochgreb, S. Diesel Engine Combustion of Biomass Pyrolysis Oils. Energy Fuels 2000, 14 (2), 260-274

(40) Shihadeh, A.; Hochgreb, S. Impact of Biomass Pyrolysis Oil Process Conditions on Ignition Delay in Compression Ignition Engines. Energy Fuels 2002, 16 (3), 552-561.

(41) Lin, C.-Y.; Chen, L.-W. Engine performance and emission characteristics of three-phase diesel emulsions prepared by an ultrasonic emulsification method. Fuel 2006, 85 (5-6), 593-600.

(42) Calabria, R.; Chiariello, F.; Massoli, P. Combustion fundamentals of pyrolysis oil based fuels. Exp. Therm. Fluid Sci. 2007, 31 (5), 413420. 
diesel feeding). The reason for the higher $\mathrm{CO}$ content with the $\mathrm{OP}-\mathrm{ABF} / \mathrm{D}$ feed should be mainly related to the low emulsion injection quality, because of the high viscosity and insufficient atomization of heavy components. It should also be mentioned that $\mathrm{CO}$ emission was affected by many other reasons, such as low combustor temperature and unadjusted generator operation parameters (e.g., fuel injection advance angle, which depressed $\mathrm{CO}$ conversion and the further combustion when the emulsion was used). ${ }^{43}$

3.9. Effect of ABF/D Emulsion Fuel on Diesel Generator Operation and Corrosion on the Injector Nozzle. Pure bio-oil cannot be compressively ignited, because of its high water content and LHV. After starting the diesel generator with diesel and operating it for $15 \mathrm{~min}$, the feed fuel was converted to pure bio-oil. The generator vibrated violently, emitted dark smoke, and shut down within $<1$ min. ${ }^{2,5}$ Pure bio-oil was unfit for being directly fed into a diesel generator when no generator modifications were made.

However, the generator could be started directly using the $\mathrm{OP}-\mathrm{ABF} / \mathrm{D}$ emulsion at room temperature with a slightly longer starting time than diesel and sustained stable running for at least 30 min with steady output power, but with more soot emission than that with a diesel feed. More-severe vibration and greater noise than that with a diesel feed, at same output power, was observed; this phenomenon may be due to the retarded combustion of some less-volatile substances that exist in the OP-ABF/D emulsion, which was hard to atomize and ignite.

At the same time, incomplete combustion of the OP-ABF/ $\mathrm{D}$ fraction easily caused coking deposition by blocking and abrasion of the injector nozzle. Some acidic substances also corroded the injector. The corrosion of the injector nozzle after 30 min of operation, using the $\mathrm{OP}-\mathrm{ABF} / \mathrm{D}$ emulsion, is indicated in Figure 6; some solid particles were formed on the nozzle and some pores were blocked by the coking that was produced. To maintain stable operation when the OP-ABF/ $\mathrm{D}$ emulsion was used in the diesel generator, the materials that comprise the injector should be changed to resistant materials, to prolong the lifetime of the diesel generator, and research regarding further upgrades of the aqueous bio-oil fraction should be underway. 9,15

\section{Conclusion}

In this study, the factors that influence the ultrasonic emulsification of aqueous bio-oil fraction/diesel and the combustion characteristics of the optimized stable, nonstratified emulsion by thermogravimetric analysis (and its emission) in a diesel generator with different power outputs were investigated. It was still hard to prepare stable $\mathrm{ABF} / \mathrm{D}$ emulsions, even by adding water to pure bio-oil to extract

(43) Qi, D. H.; Chen, H.; Matthews, R. D.; Bian, Y. Z. Combustion and emission characteristics of ethanol-biodiesel-water micro-emulsions used in a direct injection compression ignition engine. Fuel, in press (DOI: 10.1016/j.fuel.2009.06.029). (or concentrate) the water-soluble fraction as light oxygenates and the carbohydrate-derived fraction and discard the heavy organic compound fraction (lignin-derived oligomers as tar). Besides other factors such as aqueous bio-oil fraction Ba, type and amount of emulsifier, and ultrasonic time, the aqueous bio-oil fraction:diesel mass ratio primarily affected the emulsion stratification. The rough premixed $\mathrm{ABF} / \mathrm{D}$ droplet was disrupted into smaller droplets in the vicinal dispersion zone by cavitation and was further stabilized with the aid of Mix 1 emulsifier, because of their closely arranged amphiphilic molecular structure during ultrasonic emulsification. Thermodynamic analysis also indicates entropy augmentation and equilibrium tendency during ultrasonic emulsification. The polar compounds in the aqueous bio-oil fraction (such as water and oxygenates (e.g., ketones, acids, aldehydes, etc.)) were embodied in the droplets of the water-in-oil ( $\mathrm{W} / \mathrm{O})$ emulsion stably, while small amounts of esters and aromatic hydrocarbons (phenols) were solubilized in the hydrophilic micelles of the polyoxyethylene fraction. However, the solubilization ability of $\mathrm{W} / \mathrm{O}$ droplets could be reduced when a high content of carbohydrate organics was present in the aqueous bio-oil fraction and a non-closely arranged interface was formed during emulsification, which might cause the high stratification rate and less stability.

Besides the main weight loss peak at the similar low temperature of $180{ }^{\circ} \mathrm{C}$ with diesel, the high weight loss temperature at $330{ }^{\circ} \mathrm{C}$ with a relatively low intensity in the $\mathrm{ABF} / \mathrm{D}$ emulsion differential thermogravimetry (DTG) curve was due to the low content of heavy and less-volatile fractions, obtained via combustion from the aqueous bio-oil fraction. It was inferred from the lower exhaust gas temperature at output powers of $<1200 \mathrm{~W}$ and the higher $\mathrm{CO}_{2}$ emission with lower $\mathrm{O}_{2}$ emission at output powers of $>1200 \mathrm{~W}$ when the emulsion, rather than diesel, was used as feed that microexplosion could have occurred during the entire output power range with lower $\mathrm{NO}_{\mathrm{X}}$ emission. Yet, the higher $\mathrm{CO}$ content with the emulsion feed should be mainly related to the low emulsion injection quality, because of high viscosity and insufficient atomization of heavy components. An unadjusted generator operation parameter (e.g., fuel injection advance angle) may be another important reason.

It should also be mentioned that the exact process of droplet disruption due to ultrasonic treatment, especially as a result of cavitation, is not yet fully understood and it is not easy to estimate the possibility for microexplosions to happen in emulsion droplets in generator, because of the lack of characteristics. Therefore, some of the conclusions were deduced from the emulsification and combustion results, and moredetailed experiments are planned in the future.

Acknowledgment. This work was supported by National Natural Research Foundation Project (Project No. 50776092) and the National High Technology Research, Development Program of China via 863 plan (Project No. 2007AA05Z416). 